\title{
BMJ Open Socioeconomic determinants of out-of- pocket pharmaceutical expenditure among middle-aged and elderly adults based on the China Health and Retirement Longitudinal Survey
}

To cite: Du J, Yang X, Chen $\mathrm{M}$, et al. Socioeconomic determinants of out-of-pocket pharmaceutical expenditure among middle-aged and elderly adults based on the China Health and Retirement Longitudinal Survey. BMJ Open 2019;9:e024936. doi:10.1136/ bmjopen-2018-024936

- Prepublication history for this paper is available online. To view these files please visit the journal online (http://dx.doi org/10.1136/bmjopen-2018024936).

Received 21 June 2018 Revised 10 June 2019 Accepted 11 July 2019

Q Check for updates

(c) Author(s) (or their employer(s)) 2019. Re-use permitted under CC BY-NC. No commercial re-use. See rights and permissions. Published by BMJ.

${ }^{1}$ School of Health Policy and Management, Nanjing Medical University, Nanjing, China

${ }^{2}$ Creative Health Policy Research Group, Nanjing Medical University, Nanjing, China

Correspondence to Dr Zhonghua Wang; wzh04@njmu.edu.cn

\section{ABSTRACT}

Objective Out-of-pocket pharmaceutical expenditure (OOPPE) is a considerable burden for middle-aged and elderly adults due to their high prevalence of diseases, insufficient income and absence of medical insurance in China. The objective of this study was to assess the determinants of 00PPE among Chinese middle-aged and elderly adults.

Methods This is a cross-sectional study based on the China Health and Retirement Longitudinal Survey conducted in 2015. The Andersen behavioural health model was used to select the factors. Binary multivariable logistic and generalised linear regressions were both applied to examine the determinants of 00PPE.

Results Of the respondents, $15.28 \%, 5.20 \%$ and $51.35 \%$ reported an 00PPE for outpatient services, inpatient services and self-medication, respectively. The 00PPE for outpatient services, inpatient services and self-medication was US\$6.66, US\$17.93 and US\$15.32, respectively. Increased age significantly influenced the likelihood of OOPPE, and older people (aged $>65$ years) had lower 00PPE for outpatient services. Having health insurance significantly reduced the likelihood of OOPPE for outpatient and inpatient services but increased 00PPE for self-medication. In general, compared with the low-income group, higher income groups had a significantly lower likelihood of having an 00PPE, and when they did the amounts were less. Generally, middleaged and elderly people with poor self-reported health status, limitation of daily activities, and critical or chronic diseases had a significantly higher likelihood of having an 0OPPE and at a significantly higher amount.

Conclusion Policy-making efforts should focus on reducing self-medication OOPPE and alleviating its associated socioeconomic determinants to ease the economic burden of diseases among middle-aged and elderly adults in China.

\section{INTRODUCTION}

While overall health, average income and life expectancy have improved worldwide over recent decades, the quality of life in older individuals has not generally followed suit. ${ }^{1}$ Ageing on average is associated with an increased prevalence of non-communicable
Strengths and limitations of this study

- This study focused on a considerable economic burden of disease, out-of-pocket pharmaceutical expenditures (00PPEs), rather than the total cost or out-of-pocket expenditure for health services.

- The China Health and Retirement Longitudinal Survey is the first national and representative survey of Chinese middle-aged and elderly adults.

- This was the first analysis of 00PPEs among Chinese middle-aged and elderly people with chronic diseases.

- Indicators were obtained without considering the impact of household factors on 0OPPE.

chronic diseases (NCDs) and complex medical conditions, indicating that the demand for healthcare services will continue to rise. A short supply of medical specialists and inequity in resource distribution further reduce access to healthcare of the most vulnerable patients. ${ }^{23}$ In addition, older patients typically have greater healthcare and medication needs than the younger population. ${ }^{4}$ Previous studies have reported that healthcare utilisation, drug consumption and medical expenditure of elderly populations are all higher than in the general population. ${ }^{5-7}$ The most recent projections of the Organisation for Economic Co-operation and Development (OECD) regarding the impact of population ageing on public expenditures suggest that health and long-term care will account for about half of the increase in age-related social costs between 2000 and 2050. ${ }^{8}$ Such ever-increasing costs already overburden the elderly, even resulting in catastrophic health expenditure (CHE) and poverty. ${ }^{9} 10$ The definition of CHE is that a family's medical expenditure is equal to or more than $40 \%$ of the family's affordability. 
Although an equitable financial system can protect households from suffering from a CHE, ${ }^{11}{ }^{12}$ it is unable to provide all required health services for free. The out-ofpocket expenditure (OOPE) thus remains a source of CHE and poverty. ${ }^{13}$ Out-of-pocket expenditure means the direct payment of money that may or may not be later reimbursed from a third-party source. Furthermore, out-of-pocket pharmaceutical expenditure (OOPPE), in particular, accounts for a high proportion of the total OOPE. According to data obtained from OECD member states, the out-of-pocket share of the total pharmaceutical spending (41\% in 2011) is more than twice as much as the out-of-pocket share of the total healthcare spending. ${ }^{14}$ High OOPPE is a burden for people, especially the older populations whose medication use and medication expenditures have been rising faster with the increase in the prevalence of chronic diseases. ${ }^{15}$ OOPPE can be classified according to the type of medical services provided or the reimbursement policy. Sanwald and Theurl ${ }^{16}$ classified OOPPE into three types: one involving over-thecounter (OTC) medicines obtained by self-medicating individuals, another involving medicines prescribed by a physician and pharmaceuticals not covered by insurance, and a third type involving pharmaceuticals covered by insurance that only needed a copayment on dispensing. Park et al estimated the OOPPE of outpatients, ${ }^{6}$ while Tobe $e t a l^{17}$ quantified the extent to which beneficiaries of the National Health Insurance Program incurred out-ofpocket medical expenses for inpatient care. Some studies used an absolute value while others applied the relative ratio of OOPPE as a portion of family drug expenses or total household health expenditure when calculating OOPPE. ${ }^{6} 1618$

As OOPPE is a form of health outcome, it can be influenced by demographic or socioeconomic factors such as age, gender, marital status, education, health insurance and location of residence. Rovira and Habibov ${ }^{19} 20$ found that health status, economic standing, possession of health insurance and other demographic factors were strong determinants of OOPPE both in Catalonia and Tajikistan. Health insurance certainly plays an important role in pharmaceutical expenditure. Look and Arora ${ }^{21}$ examined short-term changes in prescription drug use and expenditure after implementation of the Affordable Care Act and expansion of health insurance coverage in the young adult population of the USA. They found that out-of-pocket drug expenditure subsequently decreased by $30 \%$ in this age group. ${ }^{21}$ Park et al and Kim et $a l^{67}$ compared the OOPPE between patients covered by national health insurance and medical aid recipients, reporting the OOPPE of national health insurance participants to be lower than that of medical aid recipients. Other health factors such as morbidity (especially of chronic diseases) can contribute to an increase in OOPPE. Rodbard et $a t^{22}$ found that individuals with type 2 diabetes mellitus (T2DM) had a significantly larger total number of prescriptions and higher OOPPE both annually and monthly in comparison with respondents without T2DM. Furthermore, as the number of chronic diseases increased, OOPPE of patients was also found to increase. $^{620}$

More than $30 \%$ of adults in China are over 50 years of age, and this proportion is expected to approach $40 \%$ by $2050 .^{23}$ Although China instituted universal health coverage over the last several decades, ${ }^{24}$ elderly people, as a vulnerable group, are more prone to greater medical expenditures due to a high prevalence of diseases, insufficient income and absence of social security mechanisms in this population. ${ }^{10}$ In addition, serious health conditions often cause poverty in this group. Although some prior studies have examined OOPPE worldwide, most have focused on whole adult populations rather than specific and more vulnerable groups. With the rapid ageing of the population, reducing the financial burden of elderly patients has become of vital importance in China. As the major component of OOPE, it is necessary to examine OOPPE as well as related factors among elderly adults. The aim of the present study was to calculate the OOPPE according to various types of health services and to estimate the determinants of OOPPE among middle-aged and elderly Chinese adults to provide evidence to policymakers and address the economic burden of disease associated with an ageing society.

\section{DATA AND METHODS \\ Data source}

This study used data from the China Health and Retirement Longitudinal Survey (CHARLS), conducted and directed by the National School of Development of Peking University. This survey, which included people from 150 counties in 28 provinces, constructed a high-quality, nationally representative sample of Chinese community-dwelling adults for research purposes concerning the elderly. The national baseline of the survey was conducted in 2011, and detailed information pertaining to 17708 individuals $\geq 45$ years of age from 10257 households was obtained. The follow-up study was carried out every 2 years; 15180 individuals responded to the survey in 2015. Data from 2015 were used in our study.

County-level units (counties or urban districts) were sampled directly. These counties covered 28 of 30 provinces in mainland China, excluding Tibet. Village and community units within county units were chosen with the help of the National Bureau of Statistics, using recently updated village-level population data. The sample used administrative villages (cun) in rural areas and neighbourhoods (shequ) in urban areas as primary sampling units (PSUs). Three PSUs were selected within each county-level unit, using probability proportional to size sampling, to select a total of 450 PSUs. Household units were selected from each PSU. The sampling frame was constructed using Google Earth base maps, and a computer-assisted personal interview program was then used to sample households and to conduct interviews via laptop computers. The OOPPE for outpatient, inpatient 
and self-medication was recalled by participants combined with their receipts during the health services.

The survey questionnaire collected information on demographic backgrounds, family, health status, healthcare and insurance, occupation, retirement and pension, income, expenditure, and assets. OOPPE was calculated from the provided data, and related information was extracted as well.

\section{Patient and public involvement}

On the basis of 450 PSUs, households were randomly selected by CHARLS-Geographic Information System (GIS) software. Individuals over 45 years old and their households were identified. The participants provided written informed consent before participating in the study. Patients and the public were not involved in the study analysis.

\section{Variable selection}

The independent variable OOPPE can be classified according to the type of medical service provided. For our study, we considered outpatient, inpatient and self-medicated OOPPE types. Self-medication mainly refers to the purchase of medicine in pharmacies, such as prescription medicine, OTC medicine, Chinese traditional herbs and health supplement. The definitions of the three types of OOPPE are given in table 1.

Independent variables were selected based on the Andersen healthcare utilisation model. This model has guided systematic investigations into factors that lead to use of healthcare services, including predisposing, enabling and need factors. Andersen's model has been widely used to elucidate utilisation of healthcare services since it was created in the late 1960s. ${ }^{25}$ Predisposing factors include demographics (age, gender and marital status), health beliefs (values or attitudes related to health and illness) and social structure (acculturation, immigration and literacy). In this study, predisposing factors included age, gender, marital status and education. Enabling factors included personage, household and resource characteristics permitting healthcare accessibility to individuals. Thus, household income level, individual health insurance and place of residence were considered as enabling factors in this study. Need factors were related to either perceived need for care or as diagnosed or evaluated by a healthcare professional. Perceived need was measured by self-reported health. Evaluated or diagnosed need was considered as a health status determined by physicians and involved specific indicators, such as the presence of chronic or critical disease, body mass index (BMI), limitations in activities of daily living and limitations in instrumental activities of daily living. Smoking and drinking are also included as components of need factors.

\section{Data analysis}

The OOPPE for outpatient, inpatient and self-medication was calculated based on data obtained from the questionnaires. Outpatient OOPPE was the amount spent on physician visits as reported by individuals over the past month. Inpatient OOPPE included the cost of hospitalisation reported by individuals over the past year. Self-medication OOPPE was the amount reported paid by individuals for OTC medications over the past month.

Logistic regression and multiple linear regression models were conducted to evaluate how the three kinds of factors affect the likelihood and amount of OOPPE, respectively. The likelihood of an OOPPE was dichotomised into $0=$ 'reported no OOPPE' and $1=$ 'reported OOPPE'. Amounts of OOPPE were processed using a log (U) function to avoid data non-stationarity. The regression of OOPPE for outpatient, inpatient and self-medication was conducted separately. Individual sampling weight was corrected by non-response based on household sampling weight. Individual weight and family weight were constructed by inverse probability weighting factor. All independent variables were categorised into predisposing, enabling and need factors, which were then processed as dummy variables. Dependent and independent variables are described in table 1.

The follow-up 2015 survey included a total of 15180 participants. Respondents with any missing variables were excluded to ensure an accurate analysis. A final number of 9932 participants were enrolled in this study.

\section{RESULTS}

\section{Basic information of samples}

Descriptive statistics of the samples evaluated are shown in table 2. The numbers and percentages of respondents who reported outpatient, inpatient and self-medication OOPPE were 1518 (15.28\%), $516(5.20 \%)$ and 5100 $(51.35 \%)$, respectively. Among individuals included in the survey, $27.54 \%$ were $>65$ years of age and $10.96 \%$ had received at least a secondary education. Only $4.82 \%$ did not participate in basic health insurance, and $78.29 \%$, $10.44 \%$ and $4.38 \%$ of respondents were enrolled in New Cooperative Medical Insurance (NCMI), Urban Employee Medical Insurance (UEMI) and Urban Resident Medical Insurance (URMI), respectively. Of the individuals, $76.15 \%$ were concentrated in a low-income group (US\$0-US\$1639), and nearly $65 \%$ of middleaged and elderly people lived in the countryside. Most elderly individuals surveyed did not report limitation in daily or instrumental activities of daily living. Nearly half of the middle-aged and elderly individuals surveyed had a BMI of greater than 24, signifying obesity. All three types of OOPPE (outpatient, inpatient and self-medication) revealed the sample distribution of each variable to generally follow the same trend of the overall sample distribution.

\section{OPPE for outpatient services, inpatient services and self-} medication

As shown in table 3, the OOPPEs for outpatient services, inpatient services and self-medication of the 9932 individuals were US $\$ 6.66$, US $\$ 17.93$ and US $\$ 15.32$, respectively. 
Table 1 Description for the dependent and independent variables

\section{Categories Indicators/Survey questions}

\section{Dependent variables}

OOPPE for Reported out-of-pocket expenditure for outpatient outpatient services.

The amount of OOPPE for outpatient services.

OOPPE for inpatient Reported out-of-pocket expenditure for inpatient services.

The amount of OOPPE for inpatient services.

OOPPE for self- Reported out-of-pocket expenditure for selfmedication medication.

The amount of OOPPE for self-medication.

How much will you eventually pay out-of-pocket for the medications, including prescriptions obtained during your most recent physician visits last month? How much did you pay out-of-pocket for medication costs during your most recent inpatient care last year?

How much did you pay out-of-pocket for selfmedication last month?

\section{Independent variables}

Predisposing factors

\begin{tabular}{lll} 
Age & $\leq 50 / 50-65 />65$ & \\
Gender & Male/Female & \\
Education & Less than lower secondary \\
& Upper secondary and vocational training \\
& Tertiary & What is the highest level of education you obtained? \\
Marital status & $\begin{array}{l}\text { Married/Partnered } \\
\text { Separated/Divorced/Widowed/Single }\end{array}$ & What is your marital status? \\
\hline Enabling factors &
\end{tabular}

\section{Enabling factors}

Insurance

\section{No social health insurance}

Urban Employee Medical Insurance (UEMI)

Urban Resident Medical Insurance (URMI)

New Cooperative Medical Insurance (NCMI)

Urban and Rural Resident Medical Insurance (URRMI)

$\begin{array}{ll}\text { Income } & \text { Low-income group ( } \leq \text { US\$1639) } \\ & \text { Median-income group (US\$1639-US\$8197) } \\ & \text { High-income group (>US\$8197) } \\ \text { Living } & \text { Rural/Urban }\end{array}$

Need factors

Self-reported health Very good/Good/Fair/Poor/Very Poor
ADL
Without ADL/with any ADL

IADL

Without IADL/with any IADL
Are you the policy holder/primary beneficiary of any of the types of health insurance options listed below?
Total income includes money earned by labour, pension and money provided as support by relatives over the past year.

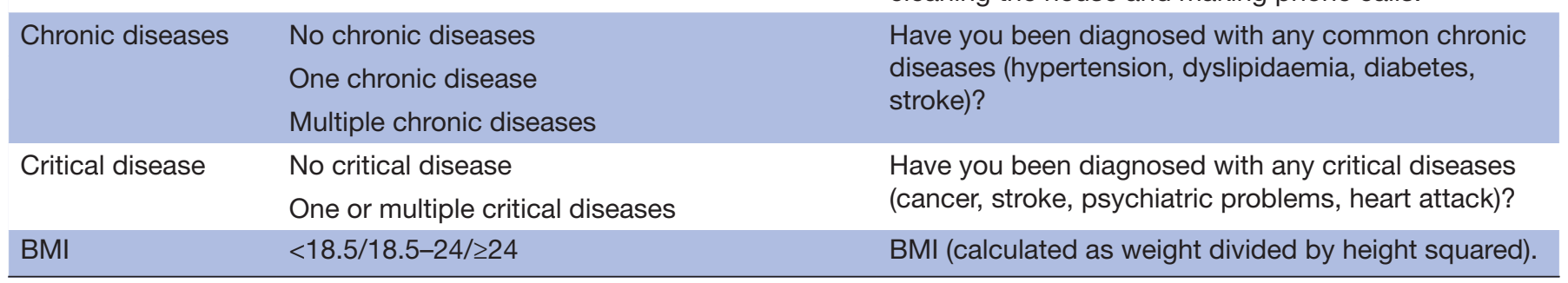

Would you say your health is very good, good, fair, poor or very poor?

Respondents reported any problems with activities including dressing, bathing, eating, getting in or out of bed, using the toilet, controlling urination and defaecation.

Respondents reported any problems with instrumental activities including managing money, taking medications, shopping for groceries, preparing meals, cleaning the house and making phone calls.

Continued 
Table 1 Continued

\begin{tabular}{lll}
\hline & Categories & Indicators/Survey questions \\
\hline Drinking & Never drank/ever drank & $\begin{array}{l}\text { Respondent reported whether they drank alcoholic } \\
\text { beverages in the past. }\end{array}$ \\
Smoking & Never smoked/ever smoked & Respondent reports whether they smoked in the past. \\
\hline
\end{tabular}

The UEMI for those who were employed in the urban sector was formally established in 1998. After this, the NCMI for rural residents as well as URMI covering urban residents who had no formal employment were introduced in 2003 and 2007 , respectively. In 2012 , the integration of URMI and NCMI was gradually carried out nationwide to form URRMI.

ADL, activities of daily living limitations; BMI, body mass index; IADL, instrumental activities of daily living limitations; OOPPE, out-of-pocket pharmaceutical expenditure.

Predisposing factor analysis revealed that individuals aged 50-65 preferred to pay for outpatient services and self-medication. The highest OOPPE for inpatient services was found to have been spent by individuals younger than 50. Compared with men, women paid slightly higher amounts for outpatient and inpatient services. Middleaged and elderly adults who had a tertiary education paid more for self-medication but less for outpatient services. Individuals who had an upper secondary and vocational training level of education paid the highest for inpatient services, the mean of which was 17.93. Compared with separated, divorced, widowed or single persons, the cost paid by married or partnered persons for outpatient services, hospitalisation and self-medication was relatively high.

Analysis of enabling factors revealed that those enrolled in URMI paid the highest OOPPE for outpatient services. The lowest amount was paid by persons enrolled in Urban and Rural Resident Medical Insurance (URRMI). Individuals enrolled in URMI paid the highest cost for hospitalisation and self-medication. The high-income group paid a greater amount of cost for outpatient services and self-medication, but the low-income group paid the highest cost for inpatient care. Urban residents paid a higher amount of cost for inpatient services and self-medication. However, they paid a slightly lower cost for outpatient care than did rural residents.

Analysis of the need factors revealed that the OOPPE for outpatient care, hospitalisation and self-medication generally increased as the level of self-reported health decreased from 'very good' to 'very poor'. The costs borne by individuals who reported their health to be poor were about four to nine times as much as those who reported excellent health. Individuals who are not restricted in daily activities paid much less money for self-medication than those who were restricted, as did persons with or without limitations in instrumental activities of daily living. Individuals with one or multiple chronic diseases paid a greater cost for outpatient care, hospitalisation and self-medication, and those who suffered from multiple chronic diseases or had critical diseases paid more for medicine than those with only one chronic disease or without critical diseases. Middle-aged and elderly adults who were obese and reported unhealthy living habits (such as drinking) also had higher cost than those who lived healthier lifestyles.

\section{Result of logistic regression on the 00PPE incidence}

Table 4 shows the results of the applied binary logistic regression on the likelihood of an OOPPE among middleaged and elderly adults. Among the predisposing factors, age significantly affected the likelihood of an OOPPE for any type of healthcare. Compared with younger middleaged people (age $\leq 50$ ), older individuals had a lower likelihood of having an OOPPE. Most enabling factors were predicted to significantly impact the likelihood of an OOPPE. Specifically, enrolment in social health insurance schemes significantly reduced the likelihood of having an OOPPE for outpatient and inpatient care among middleaged and elderly people. Furthermore, among the three kinds of social health insurance schemes available, persons enrolled in URRMI had the lowest likelihood of OOPPE $(\mathrm{OR}=0.42 ; \mathrm{OR}=0.34)$. Compared with participants in UEMI, those enrolled in resident health insurance (both NCMI and URMI) had a relatively lower likelihood of having any OOPPE. In addition, middle-aged and elderly adults with higher income were found to have a lower likelihood of any OOPPE in comparison with those in the low-income group. Apart from some life habit indicators, other need factors were all found to significantly associate with an OOPPE. Compared with those who reported their health to be 'very good', middle-aged and elderly persons with 'poor' and 'very poor' self-reported health levels were found to have a high likelihood of having an OOPPE for outpatient care and self-medication. Individuals with a self-reported health that was 'good' and 'fair' demonstrated a lower likelihood of OOPPE for inpatient services. Individuals who were limited in their daily activities and suffered from chronic diseases were found to have a higher likelihood of OOPPE for outpatient care and self-medication. Individuals afflicted with chronic or critical diseases exhibited a higher likelihood of OOPPE for outpatient care, inpatient care and self-medication, and the likelihood increased as the number of chronic diseases suffered by individuals increased. Middle-aged and elderly adults with a normal BMI were found to have a lower likelihood of OOPPE involving any types of health services. 
Table 2 Description of sample

\begin{tabular}{|c|c|c|c|}
\hline Total & $\begin{array}{l}\text { OOPPE for } \\
\text { outpatient } \\
\text { services }\end{array}$ & $\begin{array}{l}\text { OOPPE for } \\
\text { inpatient } \\
\text { services }\end{array}$ & $\begin{array}{l}\text { OOPPE } \\
\text { for self- } \\
\text { medication }\end{array}$ \\
\hline $\mathrm{N}=9932$ & $\mathrm{n}=1518$ & $n=516$ & $n=5100$ \\
\hline
\end{tabular}

Predisposing factors

Age (\%)

\begin{tabular}{|lrrrr|}
\hline$\leq 50$ & 17.91 & 17.90 & 14.53 & 17.80 \\
\hline $50-65$ & 54.55 & 54.30 & 51.94 & 55.50 \\
\hline$>65$ & 27.54 & 27.90 & 33.53 & 26.70 \\
\hline Gender (\%) & & & & \\
Male & 46.89 & 40.30 & 48.06 & 45.60 \\
\hline Female & 53.11 & 59.70 & 51.94 & 54.40 \\
\hline Education (\%) & & & \\
\hline Less than lower secondary & 89.05 & 89.70 & 87.60 & 89.30 \\
\hline Upper secondary and vocational training & 9.57 & 9.30 & 10.66 & 9.40 \\
\hline Tertiary & 1.39 & 1.10 & 1.74 & 1.30 \\
\hline Marital status (\%) & & & & 88.10 \\
\hline Married/Partnered & 87.55 & 86.60 & 87.02 & 11.90 \\
\hline Separated/Divorced/Widowed/Single & 12.45 & 13.40 & 12.98 &
\end{tabular}

Enabling factors

Insurance (\%)

No basic insurance

4.82

4.30

3.49

3.90

UEMI

10.44

9.80

10.85

10.20

URMI

4.38

3.90

4.46

4.80

NCMI

78.29

80.10

79.26

78.90

URRMI

2.06

1.90

$1.94 \%$

2.20

Income (\%)

Low-income group

76.15

79.30

80.43

77.20

Median-income group

18.63

15.70

14.92

18.40

High-income group

5.23

4.90

4.65

4.40

Living (\%)

Rural

64.86

65.20

62.79

64.30

Urban

35.14

34.80

37.21

35.70

Needing factors

Self-reported health (\%)

\begin{tabular}{lrrrr} 
Very good & 8.00 & 3.20 & 4.46 & 5.40 \\
Good & 13.95 & 5.50 & 8.14 & 11.00 \\
Fair & 53.13 & 49.10 & 40.89 & 52.70 \\
Poor & 20.18 & 34.10 & 33.72 & 24.70 \\
Very poor & 4.73 & 8.10 & 12.79 & 6.20 \\
ADL (\%) & & & & \\
Without ADL & 83.08 & 75.20 & 75.78 & 79.50 \\
With any ADL & 16.92 & 24.80 & 24.22 & 20.50 \\
IADL (\%) & & & & \\
Without IADL & 77.63 & 70.40 & 66.86 & 75.60 \\
With any IADL & 22.37 & 29.60 & 33.14 & 24.40 \\
\hline
\end{tabular}

Continued 


\begin{tabular}{|c|c|c|c|c|}
\hline & Total & $\begin{array}{l}\text { OOPPE for } \\
\text { Outpatient } \\
\text { services }\end{array}$ & $\begin{array}{l}\text { OOPPE for } \\
\text { inpatient } \\
\text { services }\end{array}$ & $\begin{array}{l}\text { OOPPE } \\
\text { for self- } \\
\text { medication }\end{array}$ \\
\hline & $\mathrm{N}=9932$ & $n=1518$ & $n=516$ & $\mathrm{n}=5100$ \\
\hline \multicolumn{5}{|l|}{ Chronic diseases (\%) } \\
\hline No chronic diseases & 60.16 & 49.70 & 42.64 & 52.40 \\
\hline One chronic disease & 27.44 & 31.40 & 31.20 & 31.30 \\
\hline Multiple chronic diseases & 12.40 & 18.80 & 26.16 & 16.40 \\
\hline \multicolumn{5}{|l|}{ Critical disease (\%) } \\
\hline No critical disease & 77.03 & 66.10 & 56.78 & 71.60 \\
\hline One or multiple critical diseases & 22.97 & 33.90 & 43.22 & 28.40 \\
\hline \multicolumn{5}{|l|}{ BMI (\%) } \\
\hline$<18.5$ & 5.88 & 6.90 & 6.59 & 5.20 \\
\hline $18.5-24$ & 48.99 & 46.00 & 46.90 & 46.50 \\
\hline$\geq 24$ & 45.13 & 47.20 & 46.51 & 48.30 \\
\hline \multicolumn{5}{|l|}{ Drinking (\%) } \\
\hline Never drank & 55.69 & 59.40 & 53.10 & 55.90 \\
\hline Ever drank & 44.31 & 40.60 & 46.90 & 44.10 \\
\hline \multicolumn{5}{|l|}{ Smoking (\%) } \\
\hline Never smoked & 57.68 & 63.90 & 58.33 & 58.30 \\
\hline Ever smoked & 42.32 & 36.10 & 41.67 & 41.70 \\
\hline
\end{tabular}

Calculations were weighted using individual sampling weights and adjusted for household and individual responses.

ADL, activities of daily living limitations; BMI, body mass index; IADL, instrumental activities of daily living limitations; NCMI, New Cooperative Medical Insurance; OOPPE, out-of-pocket pharmaceutical expenditure; UEMI, Urban Employee Medical Insurance; URMI, Urban Resident Medical Insurance; URRMI, Urban and Rural Resident Medical Insurance.

\section{Result of multiple regression model evaluation of 00PPE}

Data obtained from multiple regression model analysis of OOPPE amounts are presented in table 5 . The goodness of fit of regression of the OOPPE for outpatient, inpatient and self-medication was $0.51,0.42$ and 0.93 , respectively. Among individuals with predisposing factors, older people (age $>65$ ) were found to have a lower amount of OOPPE for outpatient care. Women had significantly higher cost for outpatient services, but lower amounts for inpatient care in comparison with men. Individuals with a higher level of education paid a greater amount of OOPPE for inpatient services and self-medication. In addition, the amount of OOPPE for self-medication was significantly higher in the married or partnered population. The presence of enabling factors revealed a significantly positive correlation between possession of social health insurance and OOPPE, implying that participation in health insurance significantly increased OOPPE. Individuals covered by the NCMI paid significantly higher amounts for all three kinds of OOPPE in comparison with those without health insurance. Individuals covered by UEMI, URMI and URRMI spent greater OOPPE on self-medication, and coefficients were estimated to be greater than those of OOPPE for outpatient or inpatient services. Compared with the low-income group, the median-income and high-income groups were significantly associated with lower amounts of OOPPE for inpatient services and self-medication. Respondents living in urban areas spent more on OOPPE involving self-medication. Persons whose self-reported health status was poor or very poor paid the highest for all three kinds of OOPPE. Individuals with physical or instrumental limitations were also significantly associated with high OOPPE. Furthermore, as chronic diseases increased in individuals, their cost for all types of healthcare increased. This effect was estimated to be greatest in OOPPE for self-medication. Individuals suffering from critical diseases were also found to have higher OOPPE. BMI also positively associated with OOPPE for self-medication. Drinkers paid significantly more OOPPE for inpatient services, while smokers paid less.

\section{DISCUSSION}

As the prevalence of NCDs in the older population increases, the demand for health utilisation also increases. As the Chinese population continues to age, CHE and impoverishment due to medical costs will become increasingly widespread. While many studies evaluated factors affecting health and out-of-pocket costs rather than OOPPE itself, especially among vulnerable groups, this study aimed to evaluate OOPPE in middle-aged 
Table 3 OOPPE among middle-aged and elderly individuals (US\$)

\begin{tabular}{llll}
$\begin{array}{l}\text { OOPPE for outpatient } \\
\text { services }\end{array}$ & $\begin{array}{l}\text { OOPPE for inpatient } \\
\text { services }\end{array}$ & & $\begin{array}{l}\text { OOPPE for self- } \\
\text { medication }\end{array}$ \\
\cline { 1 - 1 } Mean SD & Mean SD & & Mean SD
\end{tabular}

Predisposing factors

Age

$\leq 50$

6.40

39.07

24.10

440.03

12.68

42.02

50-65

6.86

82.72

120.97

16.02

52.03

$>65$

6.43

50.33

19.25

178.76

15.66

48.02

Gender

Male

6.39

84.25

17.00

131.73

14.56

50.03

Female

6.90

50.89

18.75

285.61

15.99

48.62

Education

Less than lower secondary

6.68

71.50

15.59

138.22

14.64

46.17

Upper secondary and vocational training

6.98

39.50

37.69

595.90

20.95

71.71

Tertiary

2.94

10.99

31.78

184.15

20.32

50.71

Marital status

Married/Partnered

6.69

70.93

18.39

237.36

15.62

50.53

Separated/Divorced/Widowed/Single

$\begin{array}{lll}6.42 & 49.02 & 14.71\end{array}$

130.93

13.19

39.42

Enabling factors

Insurance

No basic insurance

5.42

40.83

14.93

130.78

11.99

36.27

UEMI

7.23

48.86

20.35

150.62

22.34

65.45

URMI

7.71

41.19

60.84

873.59

23.64

73.98

NCMI

6.67

74.06

15.54

137.16

14.02

45.35

URRMI

4.06

16.86

12.00

69.86

19.22

53.61

Income

Low-income group

7.16

76.19

19.10

251.37

14.76

48.23

Median-income group

4.20

19.80

14.22

121.98

16.81

49.96

High-income group

8.16

63.24

14.01

104.49

18.22

60.61

Living

Rural

7.07

76.59

16.06

143.51

13.44

43.75

Urban

5.89

50.56

21.37

329.28

18.80

57.99

Needing factors

Self-reported health

\begin{tabular}{lrrrrrr} 
Very good & 4.47 & 58.82 & 9.28 & 73.71 & 5.07 & 17.40 \\
Good & 1.12 & 10.65 & 10.12 & 98.48 & 7.48 & 24.34 \\
\hline Fair & 4.83 & 35.09 & 12.47 & 105.87 & 12.97 & 41.85 \\
\hline Poor & 13.90 & 132.41 & 26.41 & 194.12 & 25.37 & 67.32 \\
Very poor & 16.37 & 64.93 & 80.70 & 871.68 & 39.32 & 93.15 \\
ADL & & & & & & 45.28 \\
Without ADL & 5.85 & 66.58 & 16.90 & 237.72 & 13.48 & 64.74 \\
With any ADL & 10.61 & 77.56 & 22.97 & 163.15 & 24.36 & \\
IADL & & & & & & \\
Without IADL & 5.99 & 69.20 & 16.38 & 243.99 & 13.60 & 44.47 \\
With any IADL & 8.99 & 66.34 & 23.32 & 153.04 & 21.27 & 62.86 \\
\hline Chronic diseases & & & & & & \\
\hline
\end{tabular}

Continued 


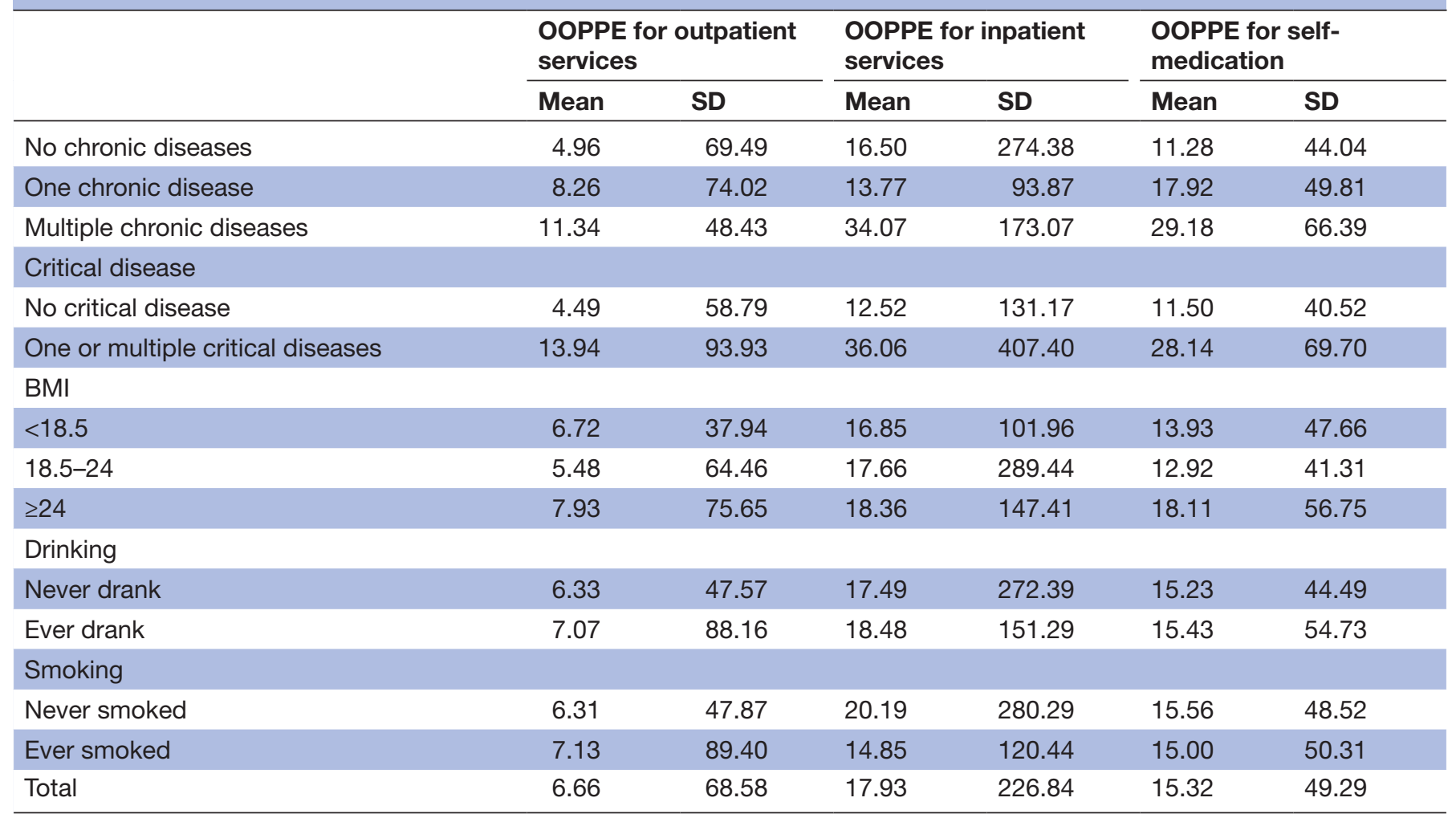

Calculations were weighted using individual sampling weights and adjusted for household and individual responses.

$\mathrm{ADL}$, activities of daily living limitations; BMI, body mass index; IADL, instrumental activities of daily living limitations; NCMI, New Cooperative Medical Insurance; OOPPE, out-of-pocket pharmaceutical expenditure; UEMI, Urban Employee Medical Insurance; URMI, Urban Resident Medical Insurance; URRMI, Urban and Rural Resident Medical Insurance.

and elderly individuals. Results suggested that socioeconomic factors influenced the likelihood and amounts of OOPPE for outpatient services, inpatient services and self-medication.

\section{OPPEs for outpatient, inpatient and self-medication have huge difference}

In this study, $15.28 \%, 5.20 \%$ and $51.35 \%$ of respondents reported an OOPPE for outpatient services, inpatient services and self-medication, and the OOPPEs were US $\$ 6.66$, US $\$ 17.93$ and US $\$ 15.32$, respectively (tables 2 and 3). Noticeable differences were likely determined by types of the health services considered for utilisation by individuals. According to the health statistics of 2015, the number of outpatient visits and hospitalisations was 6.931 billion and 0.185 billion in China in 2015. The incidence of hospitalisation was lower than that of outpatient care or self-medication. Individuals tended to choose the type of health service most in accordance with the severity of their illness and physician advice received. Most mild conditions could be treated using outpatient care or self-medication, which results in a dramatically lower proportion of OOPPE and the mean value was noted. A higher proportion and amount of OOPPE for self-medication in comparison with outpatient services might be explained by middle-aged and elderly adults preferring to use drugs based on health information available to them and perceptions concerning their conditions rather than physician advice in the setting of mild illness. Poor awareness of the importance of physician visits among this group or irrational allocation of health resources in China may have influenced such behaviour as well.

\section{Predisposing factors and 00PPE for outpatient, inpatient and self-medication}

Unlike the findings reported by prior studies, our analysis of predisposing factors revealed that older individuals were less likely to pay OOPPE (table 4). ${ }^{116} 1822$ Furthermore, elderly individuals 65 years and older paid less OOPPE for outpatient services than did younger adults (table 5). These findings may have been due to elderly individuals not making full, effective use of available health and medication resources. As the age of individuals increased, the likelihood of foregoing care rose due to advanced age, lack of insurance, insufficient income and absence of social safety nets. ${ }^{40}$ Educated, middle-aged and elderly individuals were found to pay higher OOPPE for inpatient services and self-medication (table 5), indicating that highly educated individuals had better health awareness and were better able to make fuller use of healthcare and treatment options. Married or partnered persons had a higher likelihood of self-medication OOPPE and tended to pay greater amounts (tables 4 and 5), as reported previously. ${ }^{6} 716$ 
Table 4 Logistic regression on the OOPPE among middle-aged and elderly adults

\section{OOPPE for outpatient services OOPPE for inpatient services medication

$\begin{array}{lllllll}\text { OR } & \text { SE } & \text { OR } & \text { SE } & \text { OR } & \text { SE }\end{array}$

Predisposing factors

Age, ref: $\leq 50$

\begin{tabular}{|c|c|c|c|c|c|c|}
\hline $50-65$ & $0.74^{\star \star \star}$ & 0.05 & $0.69^{* *}$ & 0.08 & $0.86^{\star *}$ & 0.05 \\
\hline$>65$ & $0.62^{\star \star \star}$ & 0.06 & $0.73^{\star \star}$ & 0.10 & $0.73^{\star \star \star}$ & 0.05 \\
\hline Gender, ref: male & $0.75^{\star \star \star}$ & 0.06 & $0.47^{\star \star *}$ & 0.06 & $0.86^{* *}$ & 0.05 \\
\hline \multicolumn{7}{|c|}{ Education, ref: less than lower secondary } \\
\hline $\begin{array}{l}\text { Upper secondary and } \\
\text { vocational training }\end{array}$ & 1.01 & 0.11 & 1.14 & 0.18 & 1.00 & 0.08 \\
\hline Tertiary & 0.76 & 0.22 & 1.24 & 0.47 & 0.95 & 0.18 \\
\hline $\begin{array}{l}\text { Marital status, ref: married/ } \\
\text { partnered }\end{array}$ & 1.03 & 0.09 & 0.89 & 0.13 & $0.87^{\star *}$ & 0.06 \\
\hline
\end{tabular}

Enabling factors

Insurance, ref: no basic insurance

$\begin{array}{lllllll}\text { UEMI } & 0.60^{\star \star \star} & 0.09 & 0.62^{\star \star} & 0.14 & 0.94 & 0.11 \\ \text { URMI } & 0.48^{\star \star \star} & 0.08 & 0.45^{\star \star} & 0.12 & 1.05 & 0.14 \\ \text { NCMI } & 0.50^{\star \star \star} & 0.05 & 0.37^{\star \star \star} & 0.05 & 0.89 & 0.07 \\ \text { URRMI } & 0.42^{\star \star \star} & 0.10 & 0.34^{\star \star} & 0.12 & 1.02 & 0.17\end{array}$

Income, ref: low-income group

$\begin{array}{lllllll}\text { Median-income group } & 0.81^{* *} & 0.08 & 0.55^{\star \star \star} & 0.09 & 0.91 & 0.06 \\ \text { High-income group } & 0.89 & 0.14 & 0.41^{\star \star \star} & 0.11 & 0.65^{\star \star \star} & 0.07 \\ \text { Living, ref: rural } & 0.98 & 0.07 & 1.04 & 0.11 & 1.04 & 0.05\end{array}$

Needing factors

Self-reported health, ref: very good

\begin{tabular}{|c|c|c|c|c|c|c|}
\hline Good & $0.43^{\star \star \star}$ & 0.06 & $0.38^{\star \star \star}$ & 0.08 & 0.97 & 0.08 \\
\hline Fair & 1.04 & 0.11 & $0.47^{\star \star \star}$ & 0.07 & $1.38^{\star \star \star}$ & 0.10 \\
\hline Poor & $1.87^{\star \star \star}$ & 0.21 & 0.85 & 0.13 & $1.95^{\star \star \star}$ & 0.16 \\
\hline Very poor & $1.69^{\star \star \star}$ & 0.25 & 1.28 & 0.25 & $2.17^{\star \star \star}$ & 0.27 \\
\hline$A D L$, ref: without ADL & $1.25^{\star \star}$ & 0.10 & 0.93 & 0.12 & $1.33^{\star \star \star}$ & 0.08 \\
\hline IADL, ref: without IADL & 1.11 & 0.08 & $1.23^{*}$ & 0.14 & 0.98 & 0.05 \\
\hline \multicolumn{7}{|c|}{ Chronic disease, ref: no chronic diseases } \\
\hline One chronic disease & $1.19^{\star \star}$ & 0.08 & $1.27^{\star *}$ & 0.14 & $1.54^{* * *}$ & 0.08 \\
\hline Multiple chronic diseases & $1.32^{\star \star}$ & 0.14 & $1.89^{\star \star \star}$ & 0.29 & $1.91^{\star \star \star}$ & 0.16 \\
\hline $\begin{array}{l}\text { Critical disease, ref: no critical } \\
\text { diseases }\end{array}$ & $1.32^{* \star \star}$ & 0.10 & $1.61^{* \star *}$ & 0.19 & $1.18^{\star \star}$ & 0.07 \\
\hline \multicolumn{7}{|l|}{ BMI, ref: $\leq 18.5$} \\
\hline $18.5-24$ & $0.49^{* \star *}$ & 0.05 & $0.43^{\star \star \star}$ & 0.06 & $0.84^{\star *}$ & 0.07 \\
\hline$>24$ & $0.49^{\star \star \star}$ & 0.05 & $0.40^{\star \star \star}$ & 0.06 & 0.94 & 0.08 \\
\hline Drinking, ref: never drank & 0.91 & 0.06 & 0.97 & 0.10 & 1.00 & 0.05 \\
\hline Smoking, ref: never smoked & $0.62^{\star \star \star}$ & 0.05 & $0.54^{\star \star \star}$ & 0.07 & $0.90^{*}$ & 0.05 \\
\hline
\end{tabular}

Estimates were weighted using individual sampling weights and adjusted for household and individual responses.

${ }^{\star * \star} \mathrm{P}<0.001,{ }^{* \star} \mathrm{p}<0.01,{ }^{*} \mathrm{p}<0.05$.

ADL, activities of daily living limitations; BMI, body mass index; IADL, instrumental activities of daily living limitations; NCMI, New Cooperative Medical Insurance; OOPPE, out-of-pocket pharmaceutical expenditure; Ref, Reference; UEMI, Urban Employee Medical Insurance; URMI,

Urban Resident Medical Insurance; URRMI, Urban and Rural Resident Medical Insurance. 
Table 5 Multiple regression model evaluation of OOPPE among middle-aged and elderly adults

\begin{tabular}{lll}
$\begin{array}{l}\text { OOPPE for outpatient } \\
\text { services }\end{array}$ & $\begin{array}{l}\text { OOPPE for inpatient } \\
\text { services }\end{array}$ & OOPPE for self-medication \\
\cline { 2 - 3 } Coefficient SE & Coefficient SE & Coefficient SE
\end{tabular}

Predisposing factors

Age, ref: $\leq 50$

\begin{tabular}{lcccccc|}
$50-65$ & -0.01 & 0.02 & 0.01 & 0.02 & 0.02 & 0.03 \\
$>65$ & $-0.04^{*}$ & 0.02 & 0.03 & 0.02 & -0.02 & 0.03 \\
Gender, ref: male & $0.04^{*}$ & 0.02 & $-0.03^{*}$ & 0.01 & $0.05^{*}$ & 0.02
\end{tabular}

Education, ref: less than lower secondary

\begin{tabular}{|c|c|c|c|c|c|c|}
\hline $\begin{array}{l}\text { Upper secondary and vocational } \\
\text { training }\end{array}$ & 0.04 & 0.03 & $0.05^{\star *}$ & 0.02 & $0.06^{*}$ & 0.03 \\
\hline Tertiary & -0.03 & 0.06 & 0.09 & 0.06 & 0.10 & 0.09 \\
\hline Marital status, ref: married/partnered & 0.00 & 0.02 & -0.02 & 0.02 & $-0.09^{\star *}$ & 0.03 \\
\hline \multicolumn{7}{|l|}{ Enabling factors } \\
\hline \multicolumn{7}{|l|}{ Insurance, ref: no basic insurance } \\
\hline UEMI & 0.05 & 0.04 & 0.06 & 0.04 & $0.22^{\star \star *}$ & 0.05 \\
\hline URMI & 0.05 & 0.05 & 0.06 & 0.04 & $0.28^{\star \star *}$ & 0.06 \\
\hline NCMI & $0.06^{\star \star}$ & 0.02 & $0.05^{\star \star}$ & 0.02 & $0.19^{\star \star \star}$ & 0.04 \\
\hline URRMI & 0.03 & 0.06 & 0.04 & 0.05 & $0.28^{\star \star \star}$ & 0.08 \\
\hline
\end{tabular}

Income, ref: low-income group

$\begin{array}{lllllll}\text { Median-income group } & 0.00 & 0.02 & -0.03^{\star} & 0.01 & 0.02 & 0.03 \\ \text { High-income group } & 0.06 & 0.04 & -0.06 & 0.04 & -0.12^{\star \star} & 0.04 \\ \text { Living, ref: rural } & 0.02 & 0.02 & 0.03^{\star} & 0.01 & 0.07^{\star \star} & 0.03\end{array}$

Needing factors

Self-reported health, ref: very good

\begin{tabular}{|c|c|c|c|c|c|c|}
\hline Good & 0.00 & 0.03 & 0.01 & 0.03 & $0.13^{\star \star \star}$ & 0.04 \\
\hline Fair & $0.14^{\star * \star}$ & 0.02 & 0.02 & 0.02 & $0.33^{\star \star \star}$ & 0.03 \\
\hline Poor & $0.35^{\star \star \star}$ & 0.03 & $0.13^{\star \star \star}$ & 0.03 & $0.60^{\star \star *}$ & 0.04 \\
\hline Very poor & $0.34^{\star \star \star}$ & 0.04 & $0.26^{\star \star \star}$ & 0.04 & $0.76^{\star \star \star}$ & 0.06 \\
\hline$A D L$, ref: without $A D L$ & $0.06^{\star \star}$ & 0.02 & -0.01 & 0.02 & $0.14^{\star \star *}$ & 0.03 \\
\hline IADL, ref: without IADL & $0.05^{\star \star}$ & 0.02 & $0.05^{\star *}$ & 0.02 & 0.02 & 0.03 \\
\hline \multicolumn{7}{|c|}{ Chronic disease, ref: no chronic diseases } \\
\hline One chronic disease & $0.04^{\star *}$ & 0.02 & 0.02 & 0.02 & $0.20^{\star * \star}$ & 0.02 \\
\hline Multiple chronic diseases & $0.06^{\star \star}$ & 0.03 & $0.10^{* \star \star}$ & 0.03 & $0.29^{\star \star \star}$ & 0.04 \\
\hline $\begin{array}{l}\text { Critical disease, ref: no critical } \\
\text { diseases }\end{array}$ & $0.12^{\star * *}$ & 0.02 & $0.08^{* \star *}$ & 0.02 & $0.18^{* * *}$ & 0.03 \\
\hline \multicolumn{7}{|l|}{ BMI, ref: $\leq 18.5$} \\
\hline $18.5-24$ & 0.00 & 0.03 & 0.01 & 0.03 & $0.13^{\star \star \star}$ & 0.04 \\
\hline$>24$ & 0.01 & 0.03 & 0.01 & 0.03 & $0.19^{\star \star \star}$ & 0.04 \\
\hline Drinking, ref: never drank & 0.00 & 0.02 & $0.03^{*}$ & 0.01 & 0.03 & 0.02 \\
\hline Smoking, ref: never smoked & -0.03 & 0.02 & $-0.03^{\star}$ & 0.01 & 0.04 & 0.03 \\
\hline
\end{tabular}

Estimates were weighted using individual sampling weights and adjusted for household and individual responses.

${ }^{* * *} \mathrm{P}<0.001,{ }^{* *} \mathrm{p}<0.01,{ }^{*} \mathrm{p}<0.05$.

ADL, activities of daily living limitations; BMI, body mass index; IADL, instrumental activities of daily living limitations; NCMI, New Cooperative Medical Insurance; OOPPE, out-of-pocket pharmaceutical expenditure; Ref, Reference; UEMI, Urban Employee Medical Insurance; URMI, Urban Resident Medical Insurance; URRMI, Urban and Rural Resident Medical Insurance. 
Enabling factors and 00PPE for outpatient, inpatient and selfmedication

Insured middle-aged and elderly individuals had a lower likelihood of OOPPE for outpatient and inpatient services (table 4), indicating that enrolment in health insurance protects participants from OOPPE and large healthcare costs, in agreement with prior studies. ${ }^{26-28}$ However, no significant effect on the likelihood of OOPPE for self-medication reflected the limited coverage provided by health insurance benefit packages in China. In addition, we found that enrolment in NCMI significantly increased OOPPE for all types of health services, as reported by Wang et $a .^{9}{ }^{9}$ However, enrolment in URMI, UEMI or URRMI alone significantly improved OOPPE for self-medication. This finding reveals that enrolment in health insurance actually resulted in a significant OOPPE increase among middle-aged and elderly adults. In particular, it is important to note that this inductive effect was relatively greater in OOPPE for self-medication among all three health insurance schemes. This also reflects that social health insurance does not significantly ease the burden of self-medication costs in China. In many cases, self-medication costs were not completely covered by health insurance or the required drugs were not considered as essential medicines by medical insurances. Middleaged and elderly individuals thus had to cover the majority of or all pharmaceutical costs themselves. Social health insurance policies should therefore be further adjusted to expand benefit packages and effectively protect individuals from drastic OOPPE for self-medication and thus effectively reduce disease burden among the elderly. The higher income group was found to have a significantly lower likelihood of paying OOPPE and lower amounts of OOPPE as well (tables 4 and 5), indicating that Chinese health insurances or other social security policies may be more inclined to protect the rich from OOPPE.

\section{Needing factors and 00PPE for outpatient, inpatient and self- medication}

Individuals with a poor self-reported health status, limitation in daily activities, and critical or chronic diseases exhibited a significantly higher likelihood of paying OOPPE and of paying a greater amount (tables 4 and 5). Poor health conditions may force this population to use health services more and thus bear greater costs for doing so. Specifically, individuals suffering from one or multiple chronic diseases had a higher likelihood of OOPPE and were likely to pay significantly more in comparison with those without any chronic illness; these findings were in agreement with previous studies. ${ }^{620}{ }^{29}$ While strategies aimed at preventing and controlling chronic diseases should be further strengthened to reduce OOPPE among middle-aged and elderly individuals, policy efforts should focus on increasing welfare subsidies and optimising benefit packages of health insurance schemes to alleviate the burden of OOPPE among individuals suffering from chronic illness. In addition, greater attention should be paid to OOPPE for self-medication among individuals suffering from chronic diseases as this has been found to exert the greatest alleviating effect on OOPPE. Middleaged and elderly adults with normal BMI values were found to have a lower likelihood of OOPPE for all types of health services. Therefore, health initiatives should focus on prevention of obesity or low weight to avoid unnecessary OOPPE.

\section{Limitations of the study}

Our findings suggest approaches to policymakers for alleviating the economic burden of diseases associated with an ageing society. However, some limitations should be taken into consideration when interpreting our findings. First, indicators were selected based on Andersen's model; however, definitions of some indicators were not entirely perfect. For example, health insurance was a kind of health insurance that patients were enrolled in rather than insurance actually used during the occurrence of health services. Such incongruence may have influenced our calculation accuracy to some extent. Second, in order to ensure the accuracy of the analysis, the samples with missing data were excluded. This will affect representation of the sample to the population to some extent. Third, even if the amount of OOPPE was acquired from the most recent medical services, recall and response bias still existed. Recall among Chinese middle-aged and elderly people is psychologically expensive. Therefore, the costs of drugs may be overestimated to some extent. Fourth, all indicators were obtained without considering the impact of household factors on OOPPE. Finally, cross-sectional data cannot be used to draw any causal conclusions about why these individuals paid OOPPE.

\section{CONCLUSION}

Although China has instituted universal health coverage and promoted its benefits over the last several decades, the incidence of OOPPE for self-medication remains much higher than OOPPE for outpatient and inpatient services. In addition, the average amount of OOPPE for self-medication is higher than that for outpatient services. Based on the Andersen healthcare utilisation model, predisposing factors such as age and enabling factors such as health insurance and income level were found to significantly affect OOPPE. Need factors, including self-reported health, multiple chronic diseases, limitations in daily activities and BMI, also significantly impacted OOPPE for health services. Policies aimed at reducing OOPPE should focus on alleviating OOPPE for self-medication and address socioeconomic and demographic factors affecting the OOPPE among middle-aged and elderly adults in China.

Acknowledgements The authors thank the National School of Development, Peking University, and other members for their support and cooperation. The authors would also like to thank Xiangjun Li who provided invaluable comments and suggestions to this paper. ZW would also like to thank the overseas research programme for outstanding young and middle-aged teachers and principals from Jiangsu Provincial Department of Education for their support. 
Contributors ZW led the study, designed the study, led the data analysis and helped in writing the draft. JD contributed to the study design and data analysis, and took the lead in writing the manuscript. XY and MC helped to sort and analyse the data and helped in writing the final draft of the manuscript. All authors read and approved the final manuscript.

Funding This study was funded by an overseas research programme for outstanding young and middle-aged teachers and principals from the Jiangsu Provincial Department of Education, and the National Natural Science Foundation of China (grant number: 71503137).

Competing interests None declared.

Patient consent for publication Not required.

Ethics approval This study was approved by the Academic Research Ethics Committee of Nanjing Medical University (reference number: 2018431). All procedures were in accordance with the ethical standards of the Helsinki Declaration.

Provenance and peer review Not commissioned; externally peer reviewed. Data availability statement Data are available in a public, open access repository. Open access This is an open access article distributed in accordance with the Creative Commons Attribution Non Commercial (CC BY-NC 4.0) license, which permits others to distribute, remix, adapt, build upon this work non-commercially, and license their derivative works on different terms, provided the original work is properly cited, appropriate credit is given, any changes made indicated, and the use is non-commercial. See: http://creativecommons.org/licenses/by-nc/4.0/.

\section{REFERENCES}

1. Gwatidzo SD, Stewart Williams J, Stewart WJ. Diabetes mellitus medication use and catastrophic healthcare expenditure among adults aged 50+ years in China and India: results from the who study on global ageing and adult health (SAGE). BMC Geriatr 2017;17:14.

2. Dall TM, Gallo PD, Chakrabarti R, et al. An aging population and growing disease burden will require ALarge and specialized health care workforce by 2025. Health Aff 2013;32:2013-20.

3. Albanese E, Liu Z, Acosta D, et al. Equity in the delivery of community healthcare to older people: findings from 10/66 dementia research Group cross-sectional surveys in Latin America, China, India and Nigeria. BMC Health Serv Res 2011;11:153.

4. Hu J, Huang C-C. Health service utilization and expenditure of the elderly in China. Asian Soc Work Pol Rev 2016;10:162-74.

5. W H L, Lee WJ, Chen LK, et al. Comparisons of annual health care utilization, drug consumption, and medical expenditure between the elderly and general population in Taiwan. Journal of Clinical Gerontology \& Geriatrics 2016;7:44-7.

6. Park E-J, Kwon J-W, Lee E-K, et al. Out-Of-Pocket medication expenditure burden of elderly Koreans with chronic conditions. Int J Gerontol 2015;9:166-71.

7. Kim S-G, Yu S-H, Park W-S, et al. [Out-of-pocket health expenditures by non-elderly and elderly persons in Korea]. J Prev Med Public Health 2005;38:408-14.

8. Gray A. Population Aging and Health Care Expenditure. China Labor Economics 2009.

9. Wang Z, Li X, Chen M, et al. Social health insurance, healthcare utilization, and costs in middle-aged and elderly community-dwelling adults in China. Int J Equity Health 2018;17.
10. Wang Z, Li X, Chen M. Catastrophic health expenditures and its inequality in elderly households with chronic disease patients in China. Int J Equity Health 2015;14:8.

11. Wagstaff A, Doorslaer EE. Equity in the finance and delivery of health care: concepts and definitions. Journal of Molecular Biology 1993;3:705-6.

12. De Graeve D, Van Ourti T, Ourti T. The Distributional impact of health financing in Europe: a review. World Econ 2003;26:1459-79.

13. Pradhan J, Dwivedi R, Banjare P. Relying on whom? correlates of out of pocket health expenditure among the rural elderly in Odisha, India. Ageing Int 2017;42:306-23.

14. OECD. Health at glance, Paris, 2013.

15. Luffman J. Out-Of-Pocket spending on prescription drugs. Perspectives on Labor \& Income 2002.

16. Sanwald A, Theurl E. Out-Of-Pocket expenditures for pharmaceuticals: lessons from the Austrian household budget survey. European Journal of Health Economics 2016;18:1-13.

17. Tobe M, Stickley A, del Rosario RB, et al. Out-Of-Pocket medical expenses for inpatient care among beneficiaries of the National health insurance program in the Philippines. Health Policy Plan 2013;28:536-48.

18. Caldbick S, Wu X, Lynch T, et al. The financial burden of out of pocket prescription drug expenses in Canada. Int $\mathrm{J}$ Health Econ Manag. 2015;15:329-38.

19. Rovira J. Determinants of out-of-pocket pharmaceutical expenditure and access to drugs in Catalonia. Applied Economics 2007;39:541-51.

20. Habibov N. Determinants of out-of-pocket expenditures on prescribed medications in Tajikistan. $J$ Health Organ Manag 2009;23:170-82.

21. Look KA, Arora P. Effects of the Affordable care act's young adult insurance expansion on prescription drug insurance coverage, utilization, and expenditures. Res Social Adm Pharm 2016;12:682-98.

22. Rodbard HW, Green AJ, Fox KM, et al. Impact of type 2 diabetes mellitus on prescription medication burden and out-of-pocket healthcare expenses. Diabetes Res Clin Pract 2010;87:360-5.

23. He W, Goodkind D, Kowal PUS. P95/16-1, an aging world: 2015. Washington, DC: U.S. Government Publishing Office, 2016.

24. Yip WC-M, Hsiao WC, Chen W, et al. Early appraisal of China's huge and complex health-care reforms. The Lancet 2012;379:833-42.

25. Jahangir E, Irazola V, Rubinstein A. Need, enabling, predisposing, and behavioral determinants of access to preventative care in Argentina: analysis of the National survey of risk factors. PLoS One 2012;7:e45053-53.

26. Galárraga O, Sosa-Rubí SG, Salinas-Rodríguez A, et al. Health insurance for the poor: impact on catastrophic and out-of-pocket health expenditures in Mexico. Eur J Health Econ 2010;11:437-47.

27. Chu T-B, Liu T-C, Chen C-S, et al. Household out-of-pocket medical expenditures and national health insurance in Taiwan: income and regional inequality. BMC Health Serv Res 2005;5:1-9.

28. Fan VY, Karan A, Mahal A. State health insurance and out-of-pocket health expenditures in Andhra Pradesh, India. Int J Health Care Finance Econ 2012;12:189-215.

29. Mclntyre D, Thiede M, Dahlgren $G$, et al. What are the economic consequences for households of illness and of paying for health care in low- and middle-income country contexts? Soc Sci Med 2006;62:858-65. 